\title{
First detection of SARS-CoV-2 B.1.617.2 (Delta) variant of concern in a symptomatic cat in Spain
}

\section{Sandra Barroso-Arévalo ( $\nabla$ sandrabarroso@ucm.es )}

Complutense University of Madrid https://orcid.org/0000-0001-6852-2487

\section{Lidia Sánchez-Morales}

Complutense University of Madrid https://orcid.org/0000-0001-7755-9812

\section{Marta Pérez-Sancho}

Complutense University of Madrid https://orcid.org/0000-0002-1493-8978

\section{Lucas Domínguez Rodríguez}

Complutense University of Madrid

Jose M Sánchez-Vizcaíno

Complutense University of Madrid

\section{Case Report}

Keywords: SARS-CoV-2, cats, pet, delta variant, transmission

Posted Date: December 1st, 2021

DOI: https://doi.org/10.21203/rs.3.rs-1124877/v1

License: (c) (i) This work is licensed under a Creative Commons Attribution 4.0 International License. Read Full License

Version of Record: A version of this preprint was published at Frontiers in Veterinary Science on April 1st, 2022. See the published version at https://doi.org/10.3389/fvets.2022.841430. 


\section{First detection of SARS-CoV-2 B.1.617.2 (Delta) variant of concern in}

\section{2 a symptomatic cat in Spain}

3 Sandra Barroso-Arévalo ${ }^{1,2}$, Lidia Sánchez-Morales ${ }^{1,2}$, Marta Pérez-Sancho ${ }^{1,2}$, Lucas

4 Domínguez ${ }^{1,2}$, José M. Sánchez-Vizcaíno ${ }^{1,2}$

$5 \quad{ }^{1}$ VISAVET Health Surveillance Center, Complutense University of Madrid, Madrid,

$6 \quad$ Spain

$7 \quad{ }^{2}$ Department of Animal Health, Faculty of Veterinary, Complutense University of

8 Madrid, Madrid, Spain

\section{Abstract}

Natural and experimental SARS-CoV-2 infection in pets has been widely evidenced since the beginning of the COVID-19 pandemic. Among the numerous affected animals, cats are one of the most susceptible species. However, little is known about viral pathogenicity and transmissibility in the case of variants of concern (VOCs) in animal hosts, such as the B.1.617.2 (Delta) variant first detected in India. Here, we have identified the B.1.617.2 (Delta) VOC in a cat living with a COVID-19 positive owner. The animal presented mild symptoms (sneezing) and a high viral load was detected in the oropharyngeal swab, suggesting that an active infection was occurring in the upper respiratory tract of the cat. Transmission from the owner to the cat occurred despite the human being fully vaccinated against SARS-CoV-2. This study documents the first detection of B.1.165.2 VOC in a cat worldwide and emphasizes the importance of performing active surveillance and genomic investigation on infected animals.

Keywords: SARS-CoV-2, cats, pet, delta variant, transmission. 


\section{Introduction}

Following the global spread of the severe acute respiratory syndrome coronavirus 2 (SARS-CoV-2), the causative agent of the disease named COVID-19, the emergence of new variants of the virus has kept the world on tenterhooks. Among the numerous variants, some of them have been proved to be even more hazardous than the original strain. These variants, denominated as variants of concern (VOCs), present higher transmission rates and a more effective evasion of the host immune system, which difficult the adequate control of the disease. The first determined VOC was the B.1.1.7 (20I/N501Y.V1), which was identified in England (Kirby, 2021). After this event, a second variant with the N501Y mutation was first detected in South Africa, named B.1.351 (20J/N501Y.V2) (Happi et al., 2021), and lately, the P.1 variant was reported in Brazil (20I/N501Y.V3) (Jia and Gong, 2021). Despite their hazardous properties, vaccination implementation helped to control the damages caused by these variants. However, since late March 2021, India started experiencing an increase in the number of COVID-19 cases reaching more than 400,000 cases and 4000 deaths reported each day in early May 2021 (European Centre for Disease Prevention and Control, 2021). These fatalities were associated with a new lineage, the B.1.617.2 (Delta) variant, that was first detected in India in December 2020 and became the most commonly reported variant in the country since mid-April 2021. The delta variant is characterized by the spike protein mutations T19R, $\Delta 157-158$, L452R, T478K, D614G, P681R, and D950N.

Several of these mutations may alter host immune response and increase viral replication, leading to higher viral loads and increased transmission rates (Johnson et al., 2020).

Although the concern regarding the delta variant in the human population is widely documented, little is known about the transmission capacity and the effects of this 
variant in animals. Due to the zoonotic origin of the COVID-19 disease, numerous experimental and field studies have been conducted in order to explore the extent of the infection in animals and to elucidate their role as reservoirs (Abdel-Moneim and Abdelwhab, 2020; Barroso-Arévalo et al., 2021a; Cleary et al., 2020; Haake et al., 2020; Hobbs and Reid, 2020). Concretely, efficient SARS-CoV-2 transmission between owners and their pets has been demonstrated worldwide (Fritz et al., 2020; Hamer et al., 2020; Ruiz-Arrondo et al., 2020). In addition, genomic surveillance in infected pets has evidenced animal infection with at least one VOC, the B.1.1.7 alpha variant (BarrosoArévalo et al., 2021b; Ferasin et al., 2021; Hamer et al., 2021). These events highlight the importance of performing genetic investigations on samples from infected pets in order to understand the transmission and evolution of the virus in these hosts.

To the best of our knowledge, here, we documented the first human to domestic cat transmission of the SARS-CoV-2 B.1.617.2 (delta) variant in Spain. Although the cat only presented subtle clinical signs, it showed high levels of viral RNA in the oropharyngeal swab taken, and virus isolation was possible from this sample. These facts evidence an active viral infection and open the question of whether the cat would act as a source of virus.

\section{Material and methods}

\subsection{Animal sampling}

Cat sampling was conducted during the owner quarantine period, 9 days after COVID19 positive confirmation of the owner. Nasal and rectal swabs, as well as feces, were collected in DeltaSwab® Virus 3ml contained in viral transport media (VTM) (Deltalab S.L., Cataluña, Spain) using protocols approved by the Complutense University of Madrid's Ethics Committee for Animal Experiments (Project License 14/2020). 
2.2 Detection of SARS-CoV-2 infection by reverse transcription-quantitative PCR (RT$q P C R)$ and virus isolation

RNA from these swabs was extracted using the KingFisher Flex System automated extraction instrument (ThermoFisher, Waltham, MA, USA), with the MagMAX

Viral/Pathogen Nucleic Acid Isolation Kit (ThermoFisher), according to the manufacturer's instructions. The detection of SARS-CoV-2 RNA was performed using the envelope protein (E)-encoding gene (Sarbeco) and two targets (IP2 and IP4) of the RNA-dependent RNA polymerase gene (RdRp) in an RT-qPCR protocol established by the World Health Organization according to the guidelines that can be found at https://www.who.int/emergencies/diseases/novel-coronavirus-2019/technicalguidance/laboratory-guidance (Corman et al., 2020).

Viral isolation was performed using the previously described methods in (Gortázar et al., 2021).

\subsection{Whole-genome sequencing and phylogenetic analysis}

Whole-genome sequence was obtained from the positive oropharyngeal sample by RTPCR with 38 primers sets following the protocol described by (Paden et al., 2020). Sequence analysis was performed using the Sequencing Analysis software v.5.3.1 (Applied Biosystems), while SeqScape v.2.5 software (Applied Biosystems) was used for sequence assembly using the SARS-CoV-2 isolate Wuhan-Hu-1, complete genome (GenBank accession number: NC_045512) as a reference genome.

Phylogenetic analysis was performed using MEGA X software (Tamura, 1992). A total of 31 representative sequences were used for the analysis, including sequences from cats and dogs, the reference genome from Wuhan, as well as variants of concern such as the B.1.1.7 variant from the United Kingdom, P.1 variant from Brazil, variant B.1.351 from South Africa, and variant B.1.617.2 from India. The final alignment included 32 
sequences and was considered adequate because it was associated with an average amino acid p-distance (1-amino acid identity) of 0.011 . This value is within the acceptance threshold of $<0.8$ (Tamura, 1992). From this alignment, the phylogenetic tree was constructed using the maximum likelihood method and Subtree-PruningRegrafting (SPR) algorithm and bootstrap testing of 2000 replicates.

Mutations were determined using the CoVsurver mutations app available on the GISAID website (https://www.gisaid.org/) (accessed on 20 October 2021). We gratefully acknowledge the various laboratories and contributors of GISAID for providing these SARS-CoV-2 sequences.

\section{Results}

\subsection{Clinical case description}

The cat, a common European 7 year old cat, was living with a confirmed COVID-19 positive owner during her whole quarantine period. The owner was fully vaccinated when became infected and presented mild symptoms of the disease. The only clinical sign of the cat reported by the owner was sneezing. The animal did not show any other symptoms during the veterinarian inspection.

\section{$3.2 R T-q P C R$ and viral isolation results}

RT-qPCR and viral isolation results are shown in Table 1.

Table 1. SARS-CoV-2 test results for a pet cat from Madrid (Spain) that was confirmed for infection with the B.1.617.2 variant of concern (VOC)

\begin{tabular}{|c|c|c|c|c|c|}
\hline \multirow{2}{*}{$\begin{array}{c}\text { Animal ID, date of } \\
\text { sample collection }\end{array}$} & \multicolumn{4}{|c|}{ RT-qPCR Ct values for swab testing } & \multirow{3}{*}{ Viral isolation } \\
\cline { 2 - 5 } & \multirow{2}{*}{ Sample type } & \multicolumn{3}{|c|}{ RT-qPCR target } & \\
\cline { 2 - 5 } & & Sarbeco & IP2 & IP4 & \\
\hline \multirow{2}{*}{$\begin{array}{c}\text { Cat_2162, October } \\
3^{\text {th }}, 2021\end{array}$} & Nasal swab & 24.09 & 24.51 & 26.24 & Negative \\
\cline { 2 - 5 } & Rectal swab & 38.6 & ND & ND & NA \\
\cline { 2 - 5 } & Faeces & ND & 39.13 & ND & NA \\
\hline
\end{tabular}


*ND: not detected; NA: not applicable

119

120

121

122

123

124

125

126

127

128

129

130

131

132

133

134

135

137

138

\subsection{Whole-genome sequencing and phylogenetic analysis}

The complete genome sequence of SARS-CoV-2 was obtained from the oropharyngeal swab from the cat (GenBank accession number: OL336792).

Evolutionary relationships among the whole genomes were inferred using maximum likelihood based on the general time-reversible model (Tamura, 1992). The analysis involved 59 nucleotide sequences, including 1st, 2nd, 3rd, and noncoding codon positions. All positions with less than $95 \%$ site coverage were eliminated. That is, fewer than 5\% alignment gaps, missing data, and ambiguous bases were allowed at any position. In the end, 29514 positions were analyzed. The bootstrap consensus tree inferred from 2000 replicates (Kumar et al., 2018) was taken to represent the evolutionary history of the taxa analyzed. Branches were collapsed if the corresponding partitions occurred in fewer than $50 \%$ of bootstrap replicates. Initial tree(s) for the heuristic search were obtained automatically by applying neighbor-joining and BioNJ algorithms to a matrix of pairwise distances estimated using maximum composite likelihood and then selecting the topology with a better log-likelihood value.

Differences in the rate of evolution among different sites were modeled using a discrete gamma distribution $(2$ categories, $+\mathrm{G}$ parameter $=0.059$ ). The resulting phylogenetic tree is shown in figure 1 . As observed in the phylogenetic tree, the genome sequence from this study (CO-2162.1) clustered with sequences belonging to the B.1.617.2 (Delta) sublineage. 


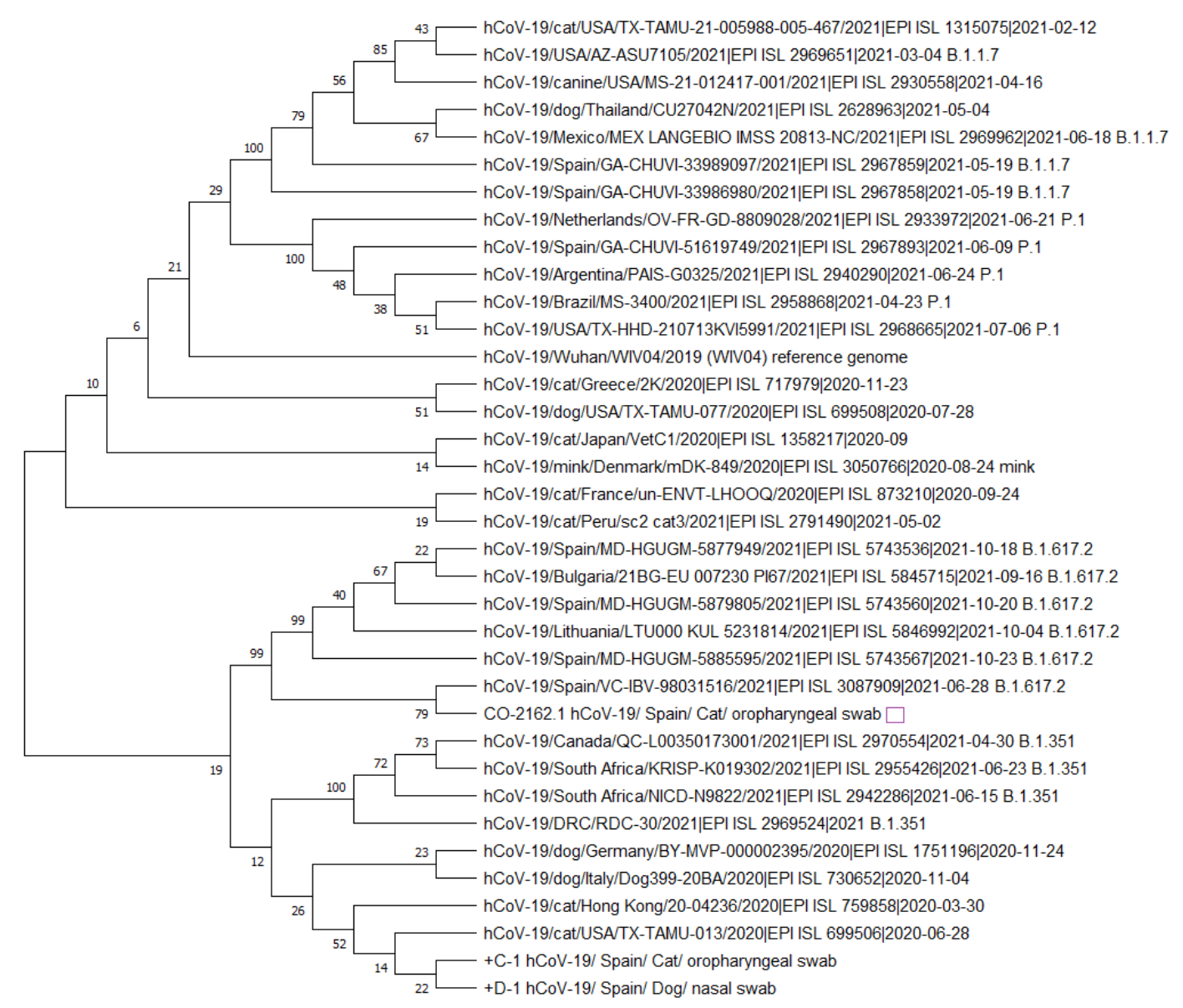

140 Figure 1. Phylogenetic analysis of SARS-CoV-2 (Severe Acute Respiratory Syndrome

141 Coronavirus 2) indicated that the whole-genome sequence from this study (purple

142 square) was similar and clustered with the SARS-CoV-2 B.1.617.2 (Delta) sublineage

143 genomes from included in the alignment.

144 Analysis in the CoVsurver mutations app (GISAID) revealed that the sequence

145 presented the following mutations: T19R(20), G142D, E156G, F157del, R158del,

146 A222V, T250I, S255F(257), L452R, T478K, D614G, P681R(674), D950N.

147 3. Discussion

148 This is, to our knowledge, the first report of the B.1.617.2 (Delta) VOC in a cat pet

149 worldwide, which confirms the transmission of this variant can occur between infected

150 people and their pets, at least in the case of cats. This variant, which is currently the 
most prevalent SARS-CoV-2 strain has shown higher transmission rates even in vaccinated people causing a decrease in vaccine effectiveness (Sanidad, 2021; Singanayagam et al., 2021). For these reasons, recent vaccine effectiveness studies now focus on this variant (Lopez Bernal et al., 2021; Pouwels et al., 2021a). All these facts evidence the importance of exploring the reach of this variant in all the possible scenarios, and, therefore, to know if this VOC can infect pets which may act as a potential source of infection. In addition, the emergence of new variants associated with animal infections cannot be dismissed, as has previously occurred in the case of minks (Oude Munnink et al., 2021; WHO, 2020).

In this study, we investigated a reverse zoonosis event in a pet cat living with a COVID19 positive owner. The cat presented light symptoms of the disease (sneezing) and was sampled during the quarantine period of its owner. Molecular analysis was performed in several samples from the cat and SARS-CoV-2 infection was confirmed by RT-qPCR, following a genomic investigation from the positive sample (oropharyngeal swab).

Thus, the phylogenetic assay revealed that the sequence from the cat presented the mutations proper of the B.1.617.2 (delta) VOC. It is also noteworthy that transmission between the infected human and the cat occurred despite the owner having a complete vaccination schedule. This finding alerts us about the high transmission capacity of this VOC, which avoided all the boundaries and jumped from the owner to the pet.

Although numerous human-based studies have demonstrated the dramatic consequences associated with infection by this VOC in humans (Mlcochova et al., 2021; Pouwels et al., 2021b), little is known about its impact on animal infection. Several field studies have previously demonstrated the presence of infection with the B.1.1.7 VOC in pets (Barroso-Arévalo et al., 2021b; Ferasin et al., 2021; Hamer et al., 2021), observing 
associated symptoms such as cardiomyopathies in some of these cases (Ferasin et al., 2021).

The delta variant has been reported to be even more transmissible than other VOCs previously detected, such as the B.1.1.7 variant (de Gier et al., 2021; Pouwels et al., 2021a). In addition, vaccine effectiveness seems to be decreased in the case of infection with this variant, as some reports have evidence (de Gier et al., 2021; Lopez Bernal et al., 2021). This fact may explain that the owner of the infected cat reported in this study was able to transmit the virus to the pet, despite being completely vaccinated. Although the virus was not isolated from the cat sample, a high viral load was detected in the oropharyngeal swab taken from the animal, which suggests that an active infection was occurring at the moment of sampling. In addition, the cat had compatible symptoms with the disease, without any known comorbidities. Taking into account that experimental studies have reflected that cats often do not present clinical signs when become infected (Bosco-Lauth et al., 2020), the presence of sneezing in this cat, suggests a higher virulence of the isolate. Under this scenario, the role of the cat as an active source of infection cannot be dismissed, as well as the potential capacity of the virus to mutate into the animal. All these facts together highlight the risk associated with pet delta variant infection and underline the importance of performing active surveillance in pets living with COVID-19 infected people, including genomic investigation in order to detect infections with VOCs or potential mutations associated with animal hosts.

\section{Acknowledgements and funding}

This research was funded by the Institute of Health Carlos III (ISCIII), project "Estudio del potencial impacto del COVID19 en mascotas y linces" (reference: COV20/01385). 
Abdel-Moneim, A.S., Abdelwhab, E.M., 2020. Evidence for SARS-CoV-2 Infection of Animal Hosts. Pathogens (Basel, Switzerland) 9, 529.

Barroso-Arévalo, S., Barneto, A., Ramos, Á.M., Rivera, B., Sánchez, R., Sánchez-Morales, L., Pérez-Sancho, M., Buendía, A., Ferreras, E., Ortiz-Menéndez, J.C., Moreno, I., Serres, C., Vela, C., Risalde, M.Á., Domínguez, L., Sánchez-Vizcaíno, J.M., 2021a. Large-scale study on virological and serological prevalence of SARS-CoV-2 in cats and dogs in Spain. Transboundary and Emerging Diseases n/a.

Barroso-Arévalo, S., Rivera, B., Domínguez, L., Sánchez-Vizcaíno, J.M., 2021b. First Detection of SARS-CoV-2 B.1.1.7 Variant of Concern in an Asymptomatic Dog in Spain. Viruses 13, 1379.

Bosco-Lauth, A.M., Hartwig, A.E., Porter, S.M., Gordy, P.W., Nehring, M., Byas, A.D., VandeWoude, S., Ragan, I.K., Maison, R.M., Bowen, R.A., 2020. Experimental infection of domestic dogs and cats with SARS-CoV-2: Pathogenesis, transmission, and response to reexposure in cats. Proceedings of the National Academy of Sciences 117, 26382.

Cleary, S.J., Pitchford, S.C., Amison, R.T., Carrington, R., Robaina Cabrera, C.L., Magnen, M., Looney, M.R., Gray, E., Page, C.P., 2020. Animal models of mechanisms of SARS-CoV-2 infection and COVID-19 pathology. Br J Pharmacol 177, 4851-4865.

Corman, V.M., Landt, O., Kaiser, M., Molenkamp, R., Meijer, A., Chu, D.K., Bleicker, T., Brünink, S., Schneider, J., Schmidt, M.L., Mulders, D.G., Haagmans, B.L., van der Veer, B., van den Brink, S., Wijsman, L., Goderski, G., Romette, J.-L., Ellis, J., Zambon, M., Peiris, M., Goossens, H., Reusken, C., Koopmans, M.P., Drosten, C., 2020. Detection of 2019 novel coronavirus (2019-nCoV) by real-time RT-PCR. Euro Surveill 25, 2000045.

de Gier, B., Andeweg, S., Backer, J.A., surveillance, R.C.-., epidemiology, t., Hahné, S.J.M., van den Hof, S., de Melker, H.E., Knol, M.J., 2021. Vaccine effectiveness against SARS-CoV2 transmission to household contacts during dominance of Delta variant (B.1.617.2), August-September 2021, the Netherlands. medRxiv, 2021.2010.2014.21264959.

European Centre for Disease Prevention and Control, C. 2021. Threat assessment brief: emergence of SARS-CoV-2 B.1.617 variants in India and situation in the EU/EEA. May 11, 2021.

Ferasin, L., Fritz, M., Ferasin, H., Becquart, P., Legros, V., Leroy, E.M., 2021. Myocarditis in naturally infected pets with the British variant of COVID-19. bioRxiv, 2021.2003.2018.435945.

Fritz, M., Rosolen, B., Krafft, E., Becquart, P., Elguero, E., Vratskikh, O., Denolly, S., Boson, B., Vanhomwegen, J., Gouilh, M.A., Kodjo, A., Chirouze, C., Rosolen, S.G., Legros, V., Leroy, E.M., 2020. High prevalence of SARS-CoV-2 antibodies in pets from COVID-19+ households. One Health 11, 100192.

Gortázar, C., Barroso-Arévalo, S., Ferreras-Colino, E., Isla, J., de la Fuente, G., Rivera, B., Domínguez, L., de la Fuente, J., Sánchez-Vizcaíno, J., 2021. Natural SARS-CoV-2 Infection in Kept Ferrets, Spain. Emerging Infectious Disease journal 27, 1994.

Haake, C., Cook, S., Pusterla, N., Murphy, B., 2020. Coronavirus Infections in Companion Animals: Virology, Epidemiology, Clinical and Pathologic Features. Viruses 12.

Hamer, S.A., Ghai, R.R., Zecca, I.B., Auckland, L.D., Roundy, C.M., Davila, E., Busselman, R.E., Tang, W., Pauvolid-Corrêa, A., Killian, M.L., Jenkins-Moore, M., Torchetti, M.K., Robbe Austerman, S., Lim, A., Akpalu, Y., Fischer, R.S.B., Barton Behravesh, C., Hamer, G.L., 2021. SARS-CoV-2 B.1.1.7 variant of concern detected in a pet dog and cat after exposure to a person with COVID-19, USA. Transboundary and Emerging Diseases n/a. Hamer, S.A., Pauvolid-Corrêa, A., Zecca, I.B., Davila, E., Auckland, L.D., Roundy, C.M., Tang, W., Torchetti, M., Killian, M.L., Jenkins-Moore, M., Mozingo, K., Akpalu, Y., Ghai, R.R., Spengler, J.R., Behravesh, C.B., Fischer, R.S.B., Hamer, G.L., 2020. Natural SARS-CoV-2 
infections, including virus isolation, among serially tested cats and dogs in households with confirmed human COVID-19 cases in Texas, USA. bioRxiv, 2020.2012.2008.416339.

Happi, A.N., Ugwu, C.A., Happi, C.T., 2021. Tracking the emergence of new SARS-CoV-2 variants in South Africa. Nat Med 27, 372-373.

Hobbs, E.C., Reid, T.J., 2020. Animals and SARS-CoV-2: Species susceptibility and viral transmission in experimental and natural conditions, and the potential implications for community transmission. Transboundary and Emerging Diseases n/a.

Jia, Z., Gong, W., 2021. Will Mutations in the Spike Protein of SARS-CoV-2 Lead to the Failure of COVID-19 Vaccines? J Korean Med Sci 36, e124.

Johnson, B.A., Xie, X., Kalveram, B., Lokugamage, K.G., Muruato, A., Zou, J., Zhang, X., Juelich, T., Smith, J.K., Zhang, L., Bopp, N., Schindewolf, C., Vu, M., Vanderheiden, A., Swetnam, D., Plante, J.A., Aguilar, P., Plante, K.S., Lee, B., Weaver, S.C., Suthar, M.S., Routh, A.L., Ren, P., Ku, Z., An, Z., Debbink, K., Shi, P.Y., Freiberg, A.N., Menachery, V.D., 2020. Furin Cleavage Site Is Key to SARS-CoV-2 Pathogenesis. bioRxiv, 2020.2008.2026.268854.

Kirby, T., 2021. New variant of SARS-CoV-2 in UK causes surge of COVID-19. Lancet Respir Med 9, e20-e21.

Kumar, S., Stecher, G., Li, M., Knyaz, C., Tamura, K., 2018. MEGA X: Molecular Evolutionary Genetics Analysis across Computing Platforms. Mol Biol Evol 35, 1547-1549.

Lopez Bernal, J., Andrews, N., Gower, C., Gallagher, E., Simmons, R., Thelwall, S., Stowe, J., Tessier, E., Groves, N., Dabrera, G., Myers, R., Campbell, C.N.J., Amirthalingam, G., Edmunds, M., Zambon, M., Brown, K.E., Hopkins, S., Chand, M., Ramsay, M., 2021. Effectiveness of Covid-19 Vaccines against the B.1.617.2 (Delta) Variant. New England Journal of Medicine 385, 585-594.

Mlcochova, P., Kemp, S.A., Dhar, M.S., Papa, G., Meng, B., Ferreira, I., Datir, R., Collier, D.A., Albecka, A., Singh, S., Pandey, R., Brown, J., Zhou, J., Goonawardane, N., Mishra, S., Whittaker, C., Mellan, T., Marwal, R., Datta, M., Sengupta, S., Ponnusamy, K., Radhakrishnan, V.S., Abdullahi, A., Charles, O., Chattopadhyay, P., Devi, P., Caputo, D., Peacock, T., Wattal, C., Goel, N., Satwik, A., Vaishya, R., Agarwal, M., Indian, S.-C.-G.C., Genotype to Phenotype Japan, C., Collaboration, C.-N.B.C.-., Mavousian, A., Lee, J.H., Bassi, J., Silacci-Fegni, C., Saliba, C., Pinto, D., Irie, T., Yoshida, I., Hamilton, W.L., Sato, K., Bhatt, S., Flaxman, S., James, L.C., Corti, D., Piccoli, L., Barclay, W.S., Rakshit, P., Agrawal, A., Gupta, R.K., 2021. SARS-CoV-2 B.1.617.2 Delta variant replication and immune evasion. Nature.

Oude Munnink, B.B., Sikkema, R.S., Nieuwenhuijse, D.F., Molenaar, R.J., Munger, E., Molenkamp, R., van der Spek, A., Tolsma, P., Rietveld, A., Brouwer, M., BouwmeesterVincken, N., Harders, F., Hakze-van der Honing, R., Wegdam-Blans, M.C.A., Bouwstra, R.J., GeurtsvanKessel, C., van der Eijk, A.A., Velkers, F.C., Smit, L.A.M., Stegeman, A., van der Poel, W.H.M., Koopmans, M.P.G., 2021. Transmission of SARS-CoV-2 on mink farms between humans and mink and back to humans. Science 371, 172.

Paden, C.R., Tao, Y., Queen, K., Zhang, J., Li, Y., Uehara, A., Tong, S., 2020. Rapid, Sensitive, FullGenome Sequencing of Severe Acute Respiratory Syndrome Coronavirus 2. Emerg Infect Dis 26, 2401-2405.

Pouwels, K.B., Pritchard, E., Matthews, P.C., Stoesser, N., Eyre, D.W., Vihta, K.-D., House, T., Hay, J., Bell, J.I., Newton, J.N., Farrar, J., Crook, D., Cook, D., Rourke, E., Studley, R., Peto, T., Diamond, I., Walker, A.S., the, C.-I.S.T., 2021a. Impact of Delta on viral burden and vaccine effectiveness against new SARS-CoV-2 infections in the UK. medRxiv, 2021.2008.2018.21262237.

Pouwels, K.B., Pritchard, E., Matthews, P.C., Stoesser, N., Eyre, D.W., Vihta, K.D., House, T., Hay, J., Bell, J.I., Newton, J.N., Farrar, J., Crook, D., Cook, D., Rourke, E., Studley, R., 
Peto, T.E.A., Diamond, I., Walker, A.S., 2021b. Effect of Delta variant on viral burden and vaccine effectiveness against new SARS-CoV-2 infections in the UK. Nat Med. Ruiz-Arrondo, I., Portillo, A., Palomar, A.M., Santibáñez, S., Santibáñez, P., Cervera, C., Oteo, J.A., 2020. Detection of SARS-CoV-2 in pets living with COVID-19 owners diagnosed during the COVID-19 lockdown in Spain: A case of an asymptomatic cat with SARS-CoV2 in Europe. Transboundary and Emerging Diseases n/a.

Sanidad, C.d.C.d.A.y.E.S.M.d., 2021. Actualización de la situación epidemiológica de las variantes de SARS-CoV-2 de preocupación (VOC) e interés (VOI) en salud pública en España.

Singanayagam, A., Hakki, S., Dunning, J., Madon, K.J., Crone, M.A., Koycheva, A., DerquiFernandez, N., Barnett, J.L., Whitfield, M.G., Varro, R., Charlett, A., Kundu, R., Fenn, J., Cutajar, J., Quinn, V., Conibear, E., Barclay, W., Freemont, P.S., Taylor, G.P., Ahmad, S., Zambon, M., Ferguson, N.M., Lalvani, A., Badhan, A., Dustan, S., Tejpal, C., Ketkar, A.V., Narean, J.S., Hammett, S., McDermott, E., Pillay, T., Houston, H., Luca, C., Samuel, J., Bremang, S., Evetts, S., Poh, J., Anderson, C., Jackson, D., Miah, S., Ellis, J., Lackenby, A., 2021. Community transmission and viral load kinetics of the SARS-CoV-2 delta (B.1.617.2) variant in vaccinated and unvaccinated individuals in the UK: a prospective, longitudinal, cohort study. The Lancet Infectious Diseases.

Tamura, K., 1992. Estimation of the number of nucleotide substitutions when there are strong transition-transversion and G+C-content biases. Mol Biol Evol 9, 678-687. 


\section{Figures}

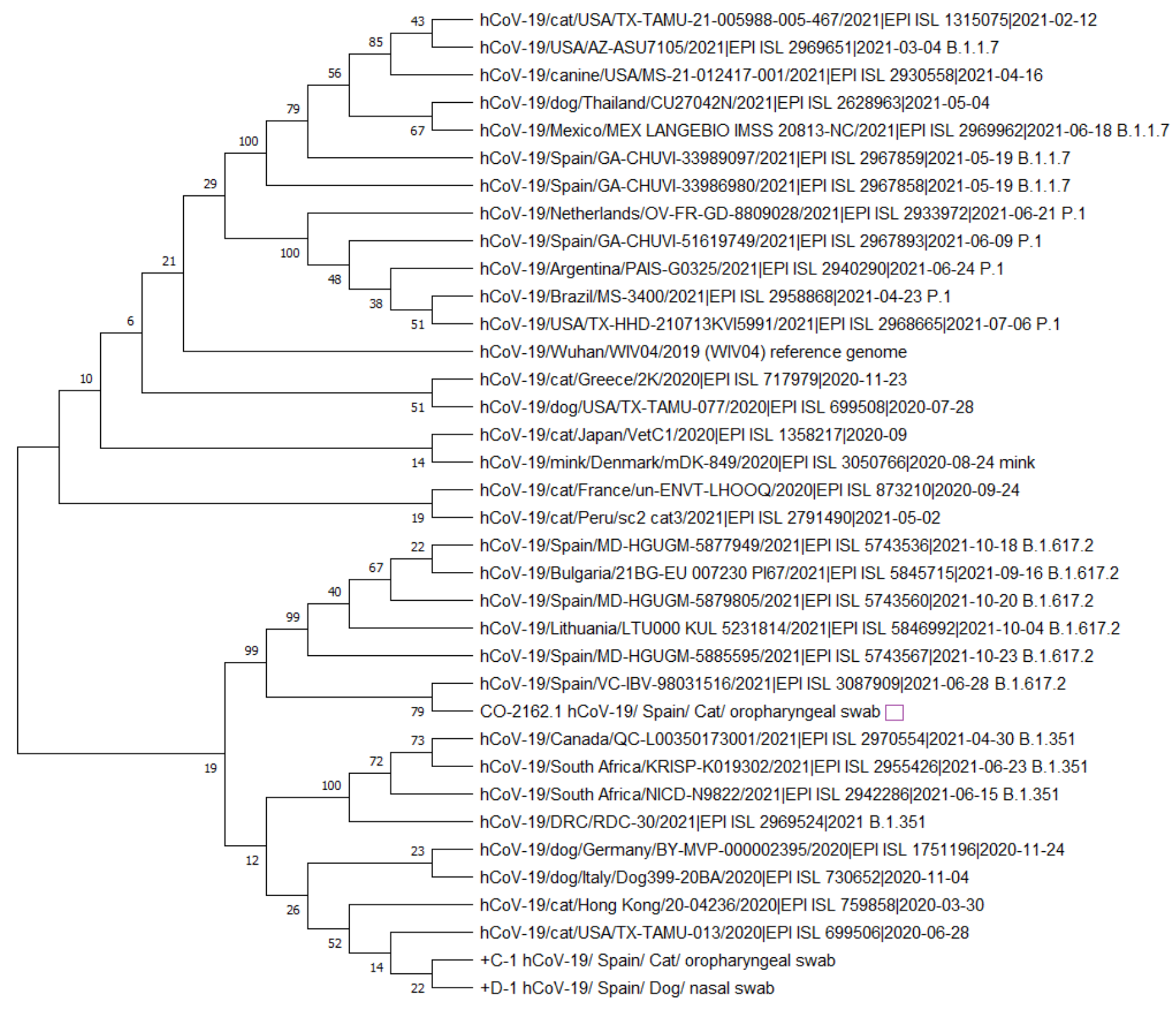

\section{Figure 1}

Phylogenetic analysis of SARS-CoV-2 (Severe Acute Respiratory Syndrome Coronavirus 2) indicated that the whole-genome sequence from this study (purple square) was similar and clustered with the SARSCoV-2 B.1.617.2 (Delta) sublineage genomes from included in the alignment. 